\title{
ASYMPTOTIC BEHAVIOR OF POSITIVE SOLUTIONS TO SEMILINEAR ELLIPTIC EQUATIONS IN $\mathbb{R}^{n}$
}

\author{
Baishun Lai, Qing Luo, and Shuqing Zhou
}

ABSTRACT. We investigate the asymptotic behavior of positive solutions to the elliptic equation

(0.1) $\quad \Delta u+|x|^{l_{1}} u^{p}+|x|^{l_{2}} u^{q}=0$ in $\mathbb{R}^{n}$

We obtain a conclusion that, for $n \geq 3,-2<l_{2}<l_{1} \leq 0$ and $q>$ $p>1$, any positive radial solution to $(0.1)$ has the following properties: $\lim _{r \rightarrow \infty} r^{\frac{2+l_{1}}{p-1}} u$ and $\lim _{r \rightarrow 0} r^{\frac{2+l_{2}}{q-1}} u$ always exist if $\frac{n+l_{1}}{n-2}<p<q, \quad p \neq$ $\frac{n+2+2 l_{1}}{n-2}, \quad q \neq \frac{n+2+2 l_{2}}{n-2}$. In addition, we prove that the singular positive solution of (0.1) is unique under some conditions.

\section{Introduction}

In this paper, we study the asymptotic behavior of positive solutions to the following equation

$$
\Delta u+K_{1}(|x|) u^{p}+K_{2}(|x|) u^{q}=0 \quad \text { in } \quad \mathbb{R}^{n}, \quad n \geq 3,
$$

and in particular, that of positive radial solutions to the equation

$$
\Delta u+|x|^{l_{1}} u^{p}+|x|^{l_{2}} u^{q}=0 \quad \text { in } \quad \mathbb{R}^{n}, \quad n \geq 3,
$$

where $-2<l_{2}<l_{1} \leq 0,1<p<q$ and $\Delta=\Sigma_{1}^{n} \frac{\partial^{2}}{\partial x_{i}^{2}}$ is the Laplace operator, and $K_{1}, K_{2}$ are locally Hölder continuous in $\mathbb{R}^{n} \backslash\{0\}$. We say a positive function $u(x)$ is an entire solution to (1.1), if $u(x)$ satisfies (1.1) pointwise in $\mathbb{R}^{n} \backslash\{0\}$.

When $K_{1}(|x|)=K_{2}(|x|), p=q$, rescaling, (1.1) reduces to

$$
\Delta u+K(|x|) u^{p}=0 \quad \text { in } \quad \mathbb{R}^{n}, \quad n \geq 3 .
$$

(1.3) originates from many mathematical and physical fields, e.g., the wellknown scalar curvature equation in the study of Riemannian geometry, the scalar field equation for the standing wave of nonlinear Schrödinger and the

Received November 11, 2009; Revised May 27, 2010.

2010 Mathematics Subject Classification. Primary 35J60; Secondary 35B05, 35B40.

Key words and phrases. semilinear elliptic equation, positive solutions, asymptotic behavior, singular solutions.

This work was supported by the Natural Science Foundation of China (10971061) and Excellent Youth Programm of Hunan Normal University (No. 080640). 
Klein-Görder equations, the Matukuma equation describing the dynamics of globular cluster of star, etc. Please refer to [16, 18, 20, 21, 23] and the references therein. There have been many works devoted to studying the existence of positive solutions of (1.3) in $\mathbb{R}^{n}$ after the first contribution by $\mathrm{Ni}$ [20] in 1982 , see $[2,6,7,8,11,12,23]$ and the references therein. One of the features of the equation is that (1.3) can posses infinitely many solutions as long as the exponent $p$ and the dimension $n$ are large enough. Recent studies in $[1,3,4,9$, 10 ] have paid special attention to this phenomenon. The purpose of this paper is to study the asymptotic behavior of positive entire solutions and to present the uniqueness result. In such perspective, we review related works as follows. In the fast decay case, i.e., $|K| \leq C r^{l}, l<-2$, Ni [20] shows that (1.3) possess infinitely many positive solutions which are bounded from below by positive constants. $\mathrm{Li}$ and $\mathrm{Ni}$ [18] shows that, for positive solution to (1.3), the limit $u_{\infty}=\lim _{x \rightarrow \infty} u(x)$ always exists. Furthermore, if $u_{\infty}=0$ for any $\varepsilon>0$, we have

$$
u(x) \leq \begin{cases}C|x|^{2-n} & \text { if } \quad p>\frac{n+l}{n-2}, \\ C_{\varepsilon}|x|^{[(1-\varepsilon)(l+2)] / 1-p} & \text { if } \quad p \leq \frac{n+l}{n-2},\end{cases}
$$

where $C_{\varepsilon}$ is a constant depending on $\varepsilon$, and if $u_{\infty}>0$, we have

$$
\left|u(x)-u_{\infty}\right| \leq \begin{cases}C|x|^{2-n} & \text { if } \quad l<-n, \\ C|x|^{2-n} \log |x| & \text { if } \quad l=-n, \\ C|x|^{2+l} & \text { if } \quad-n<l<-2,\end{cases}
$$

at $\infty$.

For the slow decay case, i.e., $K(r) \geq C r^{l}$ for $l>-2$ and $r$ being large enough, and additionally, $K(r)$ satisfying:

(K.1) $K(r)>0$ in $r>0$ and $\lim _{r \rightarrow \infty} r^{-l} K(r)=k_{\infty}>0$,

(K.2) $K(r)$ is differentiable and $\left[\frac{d}{d r}\left(r^{-l} K(r)\right]^{+} \in L^{1}, r>0\right.$,

(K.3) $K(r)$ is differentiable and $\left[\frac{d}{d r}\left(r^{-l} K(r)\right]^{-} \in L^{1}, r>0\right.$.

$\mathrm{Li}$ [17] gave an accurate description on the asymptotic behavior of positive solutions of (1.3), which can be stated as follows.

Theorem A ([17, Theorem 1]). Let $u$ be a positive radial solution of (1.3). Assume that $K$ satisfies:

(i) (K.1) and (K.2), if $0<\frac{2+l}{p-1}<\frac{n-2}{2}$ or

(ii) (K.1) and (K.3), if $\frac{n-2}{2}<\frac{2+l}{p-1}<n-2$.

Then,

$$
\lim _{r \rightarrow \infty} r^{m} u(r)=u_{\infty} \equiv\left\{\begin{array}{l}
{\left[\frac{2+l}{p-1}\left(n-2-\frac{2+l}{p-1}\right)\right]^{\frac{1}{p-1}} / k_{\infty}^{\frac{1}{p-1}} \text { or }} \\
0 .
\end{array}\right.
$$


Furthermore, if $u_{\infty}=0$, then $\lim r^{n-2} u(r)$ exists and is finite and positive.

Remark 1.1. When $l=-2$, a result similar to Theorem A holds (see $[16,17]$ ).

If $l_{1}=l_{2}=0$, the equation (1.2) reduces to the following famous LaneEmden equation

$$
\Delta u+u^{p}+u^{q}=0 \quad \text { in } \quad \mathbb{R}^{n}, n \geq 3 .
$$

The equation (1.4) has been paid much attention recently. When $p$ and $q$ are in the range $\frac{n}{n-2}<p<\frac{n+2}{n-2}<q$, the mixed growth structure (supercritical for $u$ large and subcritical for $u$ small) has a profound impact on the existence and non-existence theory, and changes the outcome for the Lane-Emden equation, the analysis is surprisingly difficult. Recently Bamon et al. ([5]) proved that if $q$ is fixed and let $p$ approach $\frac{n+2}{n-2}$ from below, then (1.4) has a large number of radial solutions. A similar result holds ([5]) for $p>\frac{n}{n-2}$ and $p$ is fixed, while letting $q$ approach $\frac{n+2}{n-2}$. In addition, they proved that (1.4) doesn't possess any solution if $q$ is fixed and then let $p$ be close enough to $\frac{n}{n-2}$.

For physical reasons, we consider the positive radial solutions to the equation (1.2), in which case it can be rewritten as

$$
u^{\prime \prime}+\frac{n-1}{r} u^{\prime}+r^{l_{1}} u^{p}+r^{l_{2}} u^{q}=0, \quad n \geq 3,
$$

where $r=|x|$.

In order to state our main results, we need some notations, which will be used throughout this paper:

$$
\begin{array}{ll}
\alpha_{1}=\frac{2+l_{1}}{p-1}, & \alpha_{2}=\frac{2+l_{2}}{q-1}, \\
\lambda_{1}^{p-1}=\alpha_{1}\left(n-2-\alpha_{1}\right), & \lambda_{2}^{q-1}=\alpha_{2}\left(n-2-\alpha_{2}\right), \\
v_{1}(r)=r^{\alpha_{1}} u(r), & v_{2}(r)=r^{\alpha_{2}} u(r),
\end{array}
$$

(note that $\alpha_{1}>\alpha_{2}, n-2-\alpha_{1}>0$ ).

We sometimes suppose

$$
p \neq \frac{n+2+2 l_{1}}{n-2}, \quad q \neq \frac{n+2+2 l_{2}}{n-2} .
$$

Now we give the following definitions:

$u(r)$ is said to be a singular solution of (1.5)(then we say $u(r)$ is singular at infinity) at infinity if $\limsup _{r \rightarrow \infty} r^{\alpha_{1}} u(r)>0$, and is said to be regular at infinitely if $\lim _{r \rightarrow \infty} r^{n-2} u$ exists. Similarly, $u(r)$ is said to be singular at 0 if $\limsup _{r \rightarrow 0} r^{\alpha_{2}} u>0$, is said to be regular at 0 if $\lim _{r \rightarrow 0} u$ exists.

One of our main results is as follows. 
Theorem 1.2. Suppose $-2<l_{2}<l_{1} \leq 0, \frac{n+l_{1}}{n-2}<p<q$, and $p, q$ satisfy (1.6). Then, for any solution of (1.5), we have:

$$
\lim _{r \rightarrow \infty} r^{\alpha_{1}} u=\lambda_{1}, \text { or } \lim _{r \rightarrow \infty} r^{n-2} u=c_{1}
$$

for some constant $c_{1}>0$. Moreover,

$$
\lim _{r \rightarrow 0} r^{\alpha_{2}} u=\lambda_{2}, \text { or } \lim _{r \rightarrow 0} u=c_{2}
$$

for some constant $c_{2}>0$.

Remark 1.3. If, say, the origin is a singularity of $u$, the term $|x|^{l_{2}} u^{q}$ is dominant and the term $|x|^{l_{1}} u^{p}$ is thus a small perturbation. At infinity, the situation is just reversed, i.e., the term $|x|^{l_{1}} u^{p}$ is dominant and the term $|x|^{l_{2}} u^{q}$ is a small perturbation.

The device in the proof of Theorem 1.2 is an energy function which plays a central role in the convergence of (1.7) and (1.8). If $p=\frac{n+2+2 l_{1}}{n-2}$, then the coefficient of the energy function is 0 and $r^{\frac{2+l_{1}}{p-1}} u$ could oscillate endlessly near the $\infty$. As the same reason, if $q=\frac{n+2+2 l_{2}}{n-2}$, then $r^{\frac{2+l_{2}}{q-1}} u$ could oscillate endlessly near the origin.

Theorem 1.4. If $-2<l_{2}<l_{1} \leq 0, \frac{n+l_{1}}{n-2}<p<q$, let $u$ be a positive radial solution of $(1.2)$, then

(i) if $q=\frac{n+2+2 l_{2}}{n-2}$, then either (1.8) holds, or $v_{2}(r)$ oscillates endlessly near the origin between two sequences $\mu_{1, i}$ and $\mu_{2, i}$ satisfying $0<\mu_{1, i}<\mu_{2, i}$ and

$$
\begin{gathered}
\lim _{i \rightarrow \infty} \mu_{1, i}=\mu_{1}, \quad \lim _{i \rightarrow \infty} \mu_{2, i}=\mu_{2}, \\
\mu_{1}=\lim _{r \rightarrow 0} \inf r^{\frac{n-2}{2}} u(r)<\lim _{r \rightarrow 0} \sup r^{\frac{n-2}{2}} u(r)=\mu_{2},
\end{gathered}
$$

where $\mu_{1}$ and $\mu_{2}$ are fixed values satisfying

(1.9) $0<\mu_{1} \leq \lambda_{2} \leq \mu_{2}, \quad b\left(\mu_{1}\right)=b\left(\mu_{2}\right)<0$ with $b(v)=\frac{1}{q+1} v^{q+1}-\frac{\lambda_{2}^{q-1}}{2} v^{2}$;

(ii) if $p=\frac{n+2+2 l_{1}}{n-2}$, then either (1.7) holds or $v_{1}(r)$ oscillates endlessly near $\infty$ between two sequences $\mu_{1, i}^{\prime}$ and $\mu_{2, i}^{\prime}$ satisfying $0<\mu_{1, i}^{\prime}<\mu_{2, i}^{\prime}$ and

$$
\begin{gathered}
\lim _{i \rightarrow \infty} \mu_{1, i}^{\prime}=\mu_{1}^{\prime}, \quad \lim _{i \rightarrow \infty} \mu_{2, i}^{\prime}=\mu_{2}^{\prime}, \\
\mu_{1}^{\prime}=\lim _{r \rightarrow \infty} \inf r^{\frac{n-2}{2}} u(r)<\lim _{r \rightarrow \infty} \sup r^{\frac{n-2}{2}} u(r)=\mu_{2}^{\prime},
\end{gathered}
$$

where $\mu_{1}^{\prime}$ and $\mu_{2}^{\prime}$ are fixed values satisfying

(1.10) $0<\mu_{1}^{\prime} \leq \lambda_{1} \leq \mu_{2}^{\prime}, b_{1}\left(\mu_{1}^{\prime}\right)=b_{1}\left(\mu_{2}^{\prime}\right)$ with $b_{1}(v)=\frac{1}{p+1} v^{p+1}-\frac{\lambda_{1}^{p-1}}{2} v^{2}$. 
For the equation (1.3), when $K \equiv 1$, it becomes the simplest model which is called generalized Lane-Emden equation or Emden-Fowler equation in astrophysics

$$
\Delta u+u^{p}=0 \quad \text { in } \mathbb{R}^{n}, \quad n \geq 3 .
$$

For (1.11), Serrin and Zhou [22] obtained the following uniqueness result.

Theorem B ([22]). If $\frac{n}{n-2}<p \leq \frac{n+2}{n-2}$, then (1.11) admits exactly one solution $\frac{2}{p-1}\left(n-2-\frac{2}{p-1}\right) r^{-\frac{2}{p-1}}$, singular at the infinity. If $p>\frac{n+2}{n-2}$, then (1.11) admits exactly one solution $\frac{2}{p-1}\left(n-2-\frac{2}{p-1}\right) r^{-\frac{2}{p-1}}$, singular at the origin.

Remark 1.5. From Theorem B, we know that (1.11) has only one solution which is singular at infinity for $\frac{n}{n-2}<p \leq \frac{n+2}{n-2}$ and singular at the origin for $p>\frac{n+2}{n-2}$. Inspired by Theorem B, we derive the following result.

Theorem 1.6. (i) If $-2<l_{2}<l_{1} \leq 0, \frac{n+l_{1}}{n-2}<p<q<\frac{n+2+2 l_{2}}{n-2}$, then (1.5) admits exactly one positive solution singular at infinity. This solution has the following exact limits:

$$
\lim _{r \rightarrow \infty} r^{\alpha_{1}} u=\lambda_{1}, \quad \lim _{r \rightarrow 0} r^{\alpha_{2}} u=\lambda_{2}
$$

(ii) If $-2<l_{2}<l_{1} \leq 0, \frac{n+2+2 l_{1}}{n-2}<p<q$, then (1.5) admits exactly one positive solution singular at the origin. This solution has the exact limits:

$$
\lim _{r \rightarrow \infty} r^{\alpha_{1}} u=\lambda_{1}, \quad \lim _{r \rightarrow 0} r^{\alpha_{2}} u=\lambda_{2} .
$$

This paper is organized as follows. In Section 2, we make some basic observations and fundamental estimates of positive solutions. In Section 3, the asymptotic behaviors at $\infty$ and 0 of positive solutions are studied. Finally, the uniqueness result is established.

Throughout this paper, unless otherwise stated, the letter $C$ will always denote various generic constant which is independent of $u$ and may change its value from line to line.

\section{Preliminaries}

In this section we present some preliminary results for radial solution $u(r)$ of (1.5), where $r=|x|$ is the radius.

Now we prove the following priori estimates, which are inspired by the work of $\mathrm{Ni}[20]$.

Lemma 2.1. If $-2<l_{2}<l_{1} \leq 0, \frac{n+l_{1}}{n-2}<p<q$, let $u(r)$ be a positive radial solution of (1.5) for $r \in(0, \infty)$, then we have, for some positive constant $C$,

(i) If $u$ tends to $\infty$ as $r \rightarrow 0$, then $u(r) \leq \mathrm{Cr}^{-\frac{2+l_{2}}{q-1}}$;

(ii) If $u$ tends to 0 as $r \rightarrow \infty$, then $u(r) \leq C r^{-\frac{2+l_{1}}{p-1}}$. 
Proof. (i) For a radial solution $u=u(r)$, we rewrite (1.5) in the following form:

$$
\left(r^{n-1} u^{\prime}\right)^{\prime}+r^{n-1}\left(r^{l_{1}} u^{p}+r^{l_{2}} u^{q}\right)=0 .
$$

Since $u \rightarrow \infty$ as $r \rightarrow 0$, there exists small $r_{0}>0$ such that $u^{\prime}<0$ in $\left(0, r_{0}\right)$.

Integrating (2.1) from $\bar{r}$ to $r\left(\bar{r}<r<r_{0}\right)$, we obtain

$$
r^{n-1} u^{\prime}(r)=\bar{r}^{n-1} u^{\prime}(\bar{r})-\int_{\bar{r}}^{r} s^{n-1}\left(s^{l_{1}} u^{p}+s^{l_{2}} u^{q}\right) d s .
$$

Therefore, $r^{n-1} u^{\prime}(r) \leq-\int_{\bar{r}}^{r} s^{n-1}\left(s^{l_{1}} u^{p}+s^{l_{2}} u^{q}\right) d s$ for all $0<\bar{r}<r$. Then letting $\bar{r} \rightarrow 0$, we obtain

$$
\begin{aligned}
r^{n-1} u^{\prime}(r) & \leq-\int_{0}^{r} s^{n-1}\left(s^{l_{1}} u^{p}+s^{l_{2}} u^{q}\right) d s \\
& \leq-\int_{0}^{r} s^{n-1} s^{l_{2}} u^{q} d s .
\end{aligned}
$$

Since $u$ is decreasing near $r=0$, we find that

$$
r^{n-1} u^{\prime}(r)<-u^{q}(r) \int_{0}^{r} s^{n-1} s^{l_{2}} d s=-\frac{1}{n+l_{2}} r^{n+l_{2}} u^{q}(r),
$$

which in turn leads to

$$
\frac{u^{\prime}(r)}{u^{q}(r)} \leq-\frac{C}{n+l_{2}} r^{l_{2}+1} .
$$

Integrating $(2.2)$ over $(\bar{r}, r)$, we have

$$
\int_{\bar{r}}^{r} \frac{u^{\prime}(s)}{u^{q}} d s \leq-C \int_{\bar{r}}^{r} s^{l_{2}+1} d s .
$$

It follows from (2.3) that

$$
u^{1-q}(r) \geq u(\bar{r})^{1-q}+\frac{q-1}{l_{2}+2}\left(r^{l_{2}+2}-\bar{r}^{l_{2}+2}\right) .
$$

Letting $\bar{r} \rightarrow 0$, we have

$$
u^{1-q}(r) \geq C r^{2+l_{2}} .
$$

So we have $u(r) \leq C r^{-\frac{2+l_{2}}{q-1}}$ for $0<r<r_{0}$, and the proof is completed.

Part (ii) of Lemma 2.1 may be handled in a similar way. Similar to the proof of (i) there exists a large number $R>0$ such that, for all $r>R$,

$$
r^{n-1} u^{\prime}(r)<-\int_{R}^{r} s^{n-1} s^{l_{1}} u^{p} d s .
$$

By a similar computation, we have

$$
\frac{u^{\prime}(r)}{u^{p}} \leq \frac{1}{n+l_{1}}\left(R^{n+l_{1}} r^{1-n}-r^{1+l_{1}}\right) .
$$

Integrating (2.4) over $(R, r)$, we have

$$
u^{1-p}(r) \geq C r^{2+l_{1}} \text { at } r=\infty .
$$


So we have $u(r) \leq C r^{-\frac{2+l_{1}}{p-1}}$ as $r \rightarrow \infty$, and the proof of part (ii) is completed.

Lemma 2.2. Let $u$ be a positive solution of (1.5). Then there exist two positive numbers $\bar{r}$ and $\overline{\bar{r}}$ such that

(i) $\left|u^{\prime}(r)\right| \leq C r^{-\left(\frac{2+l_{2}}{q-1}+1\right)} ; \quad\left|u^{\prime \prime}(r)\right| \leq C r^{-\left(\frac{2+l_{2}}{q-1}+2\right)}$ for $0<r<\bar{r}$.

(ii) $\left|u^{\prime}(r)\right| \leq C r^{-\left(\frac{2+l_{1}}{p-1}+1\right)} ; \quad\left|u^{\prime \prime}(r)\right| \leq C r^{-\left(\frac{2+l_{1}}{p-1}+2\right)}$ for $r>\overline{\bar{r}}$.

Proof. (i) Integrate (1.2) in a small ball $B_{r}$ with radius $r$ centered at 0 . From Lemma 2.1 and Green's identity, we obtain

$$
\begin{aligned}
-\omega_{n} r^{n-1} u^{\prime}(r) & =-\int_{B_{r}} \Delta u=\int_{B_{r}}\left(|x|^{l_{1}} u^{p}+|x|^{l_{2}} u^{q}\right) d x \\
& \leq C \int_{0}^{r} s^{l_{2}} u^{q} s^{n-1} d s \leq C \int_{0}^{r} s^{-\frac{2+l_{2}}{q-1} q+l_{2}+n-1} d s \\
& =C r^{-\frac{2+l_{2}}{q-1} q+l_{2}+n} .
\end{aligned}
$$

So as $r \rightarrow 0$, we have

$$
\begin{aligned}
& -u^{\prime}(r)=\left|u^{\prime}(r)\right| \leq C r^{-\frac{2+l_{2}}{q-1} q+l_{2}+1}=C r^{-\left(\frac{2+l_{2}}{q-1}+1\right)}, \\
& \left|u^{\prime \prime}\right| \leq \frac{n-1}{r}\left|u^{\prime}\right|+r^{l_{1}} u^{p}+r^{l_{2}} u^{q} \\
& \quad \leq C\left[r^{-\left(\frac{2+l_{2}}{q-1}+2\right)}+r^{l_{2}-\frac{\left(2+l_{2}\right) q}{q-1}}\right] \leq C r^{-\left(\frac{2+l_{2}}{q-1}+2\right)} .
\end{aligned}
$$

And the proof of (i) is completed.

Part (ii) of Lemma 2.2 may be handled in a similar fashion. As in the proof of (i), for large $r$ we have

$$
\begin{aligned}
\omega_{n} r^{n-1}\left(-u^{\prime}(r)\right) & =-\int_{B_{r}} \Delta u=\int_{B_{r}} s^{l_{1}} u^{p}+s^{l_{2}} u^{q} d x \\
& \leq C+C \int_{R_{0}}^{r} s^{-\frac{2+l_{1}}{p-1} p+l_{1}+n-1} d s
\end{aligned}
$$

where $R_{0}$ is a large positive number. By a simple computation, we obtain

$$
\left|u^{\prime}(r)\right| \leq C r^{-\left(\frac{2+l_{1}}{p-1}+1\right)}, \quad\left|u^{\prime \prime}(r)\right| \leq C r^{-\left(\frac{2+l_{1}}{p-1}+2\right)} \quad \text { at } r=\infty,
$$

and the proof is over.

Lemma 2.3. Suppose that $u$ is a positive solution of (1.5). Let $v(r)=r^{\alpha} u(r)$. Then $v$ satisfies

$$
v^{\prime \prime}+\frac{n-1-2 \alpha}{r} v^{\prime}-\frac{(n-2-\alpha) \alpha}{r^{2}} v+r^{l_{1}-(p-1) \alpha} v^{p}+r^{l_{2}-(q-1) \alpha} v^{q}=0 .
$$

Let $\alpha=\alpha_{1}$. Then we have $\left(v_{1}=r^{\alpha_{1}} u\right)$

$$
v_{1}^{\prime \prime}+\frac{n-1-2 \alpha_{1}}{r} v_{1}^{\prime}-\frac{\left(n-2-\alpha_{1}\right) \alpha_{1}}{r^{2}} v_{1}+\frac{v_{1}^{p}}{r^{2}}+r^{l_{2}-(q-1) \alpha_{1}} v_{1}^{q}=0 .
$$


Let $\alpha=\alpha_{2}$. Then we have $\left(v_{2}=r^{\alpha_{2}} u\right)$

$$
v_{2}^{\prime \prime}+\frac{n-1-2 \alpha_{2}}{r} v_{2}^{\prime}-\frac{\left(n-2-\alpha_{2}\right) \alpha_{2}}{r^{2}} v_{2}+\frac{v_{2}^{q}}{r^{2}}+r^{l_{1}-(p-1) \alpha_{2}} v_{2}^{p}=0 .
$$

This lemma can be proved by straight forward calculations, thus we omit it here.

Lemma 2.4. We have

$$
v_{1}^{\prime 2} r \in L^{1}(R, \infty), \quad v_{2}^{\prime 2} r \in L^{1}(0, R)
$$

where $R$ is a large positive number.

Proof. Multiplying (2.5) by $v_{1}^{\prime} r^{2}$ and integrating from $R$ to $r>R$, we obtain

$\left.\frac{v_{1}^{\prime 2} s^{2}}{2}\right|_{R} ^{r}+c^{1} \int_{R}^{r} v_{1}^{\prime 2} s d s-\left.\frac{\lambda_{1}^{p-1}}{2} v_{1}^{2}\right|_{R} ^{r}+\left.\frac{1}{p+1} v_{1}^{p+1}\right|_{R} ^{r}+\int_{R}^{r} s^{l_{2}-\alpha_{1}(q-1)+2} v_{1}^{\prime} v_{1}^{q} d s=0$, where $c^{1}=n-2-2 \alpha_{1}$.

From (ii) of Lemma 2.2, we obtain

$$
\left|v_{1}^{\prime}\right| \leq \frac{C}{r} ; \quad\left|v_{1}^{\prime \prime}\right| \leq \frac{C}{r^{2}} \text { at } r=\infty
$$

so $\left.v_{1}^{p+1}\right|_{R} ^{r},\left.\frac{v_{1}^{\prime 2} s^{2}}{2}\right|_{R} ^{r}$ and $\int_{R}^{r} s^{l_{2}-\alpha_{1}(q-1)+2} v_{1}^{\prime} v_{1}^{q} d s$ are bounded at $r=\infty$, which imply

$$
\int_{R}^{r} v_{1}^{\prime 2} s d s \leq C \text { for all } r>R
$$

since $c^{1} \neq 0$ by (1.6), and the first one (named as $\left.(2.7)_{1}\right)$ of $(2.7)$ follows. $(2.7)_{2}$ can be handled by a similar way, we omit it here.

Lemma 2.5. We have

$$
\lim _{r \rightarrow \infty} r v_{1}^{\prime}=0 ; \quad \lim _{r \rightarrow 0} r v_{2}^{\prime}=0 .
$$

Proof. Now we prove $(2.10)_{1}$. Suppose for contradiction that it is not true, then there exists a sequence $r_{k} \rightarrow+\infty$ such that

$$
\left|v_{1}^{\prime}\left(r_{k}\right) r_{k}\right| \geq C \text {. }
$$

From (2.9), one obviously has, near $\infty$,

$$
\left|\left(v_{1}^{\prime 2} r^{2}\right)^{\prime}\right| \leq \frac{M}{r}
$$

for some $M>0$. Combining the above two inequalities yields

$$
\left|v_{1}^{\prime 2}(r) r^{2}-v_{1}^{\prime}\left(r_{k}\right)^{2} r_{k}^{2}\right| \leq M\left|r-r_{k}\right| \max \left(\frac{1}{r}, \frac{1}{r_{k}}\right),
$$

and so

$$
v_{1}^{\prime 2}(r) r^{2} \geq \frac{C^{2}}{2}, r \in\left[(1+\varepsilon)^{-1} r_{k},(1+\varepsilon) r_{k}\right], \quad \varepsilon(1+\varepsilon)=\frac{C^{2}}{2 M} .
$$


This contradicts $(2.7)_{1}$.

The proof of $(2.10)_{2}$ may be handled similarly. As in the previous proof, we have, near 0 ,

$$
\left|\left(v_{2}^{\prime 2} r^{2}\right)^{\prime}\right| \leq \frac{M}{r}
$$

for some $M>0$, and by a similar calculation, we will obtain a contradiction and the proof is completed.

Lemma 2.6. Let $u$ be a positive superharmonic function near $\infty$ and $\bar{u}$ be its spherical mean. Then, $r^{n-2} \bar{u}$ is increasing as $r \rightarrow \infty$.

Proof. Put $f(t):=r^{n-2} \bar{u}(r), t=\log r$. Then $f$ satisfies

$$
f^{\prime \prime}-(n-2) f^{\prime} \leq 0
$$

and $f^{\prime}(t) \leq e^{(n-2)(t-T)} f^{\prime}(T)$ on $[T, t]$ for $T$ large. Because $f$ is positive, $f$ must be increasing near $\infty$. It implies that $\left(r^{n-2} \bar{u}(r)\right)_{r}>0$ near $\infty$.

\section{Asymptotic behavior}

In this section we investigate the asymptotic behavior at $\infty$ and 0 of positive solutions of (1.5). We prove that any positive solution of (1.5) must behave either like $r^{-\alpha_{1}}$ or $r^{2-n}$ at $\infty$. In addition, if any positive radial solution of (1.2) is singular at the origin, then it must behave like $r^{-\alpha_{2}}$. Now we give the proof of Theorem 1.2.

The proof of Theorem 1.2. Consider the function $a(r)=\frac{v_{1}^{p+1}}{p+1}-\frac{\lambda_{1}^{p-1} v_{1}^{2}}{2}$. By Lemma 2.4 and Lemma 2.5, we have, for fixed $R>0$,

$$
\frac{v_{1}^{\prime 2} r^{2}}{2} \rightarrow 0, \int_{R}^{r} v_{1}^{\prime 2} s \rightarrow c_{2}, \int_{R}^{r} r^{l_{2}-\frac{\left(2+l_{1}\right)(q-1)}{p-1}+2} v_{1}^{\prime} v_{1}^{p} \rightarrow c_{3} \text { as } r \rightarrow \infty
$$

for some constants $c_{2}$ and $c_{3}$. This implies, by (2.8), that $a(r)$ must tend to a finite constant $c_{4}$ as $t \rightarrow \infty$. We claim $v_{1}$ approaches a finite limit as $r \rightarrow \infty$. If not, we may choose two sequences $\left\{\eta_{i}\right\}$ and $\left\{\xi_{i}\right\}$ going to $\infty$ as $i \rightarrow \infty$ such that

$$
\left\{\begin{array}{l}
\left\{\eta_{i}\right\} \text { are local minima of } v_{1},\left\{\xi_{i}\right\} \text { are local maxima. } \\
\eta_{i}<\xi_{i}<\eta_{i+1}, i=1,2, \ldots
\end{array}\right.
$$

And we have

$$
v_{1}\left(\eta_{i}\right) \rightarrow m_{1}, \quad v_{1}\left(\xi_{i}\right) \rightarrow m_{2} \quad \text { as } i \rightarrow \infty
$$

for some positive constants $m_{1}, m_{2}$ with $m_{1}<m_{2}$. Since $a(r)$ tend to a finite constant as $r \rightarrow \infty$, we have

$$
\frac{m_{1}^{p+1}}{p+1}-\frac{\lambda_{1}^{p-1} m_{1}^{2}}{2}=\frac{m_{2}^{p+1}}{p+1}-\frac{\lambda_{1}^{p-1} m_{2}^{2}}{2}=c_{4},
$$


where $c_{4}$ is a constant. However, the intermediate value theorem shows that there exists $r_{i} \in\left(\eta_{i}, \xi_{i}\right)$ such that

$$
v\left(r_{i}\right)=m_{0}, \quad m_{1}<m_{0}<m_{2} \text { and } \frac{d a(v)}{d v}\left(r_{i}\right)=0 .
$$

Furthermore, we have $\frac{m_{0}^{p+1}}{p+1}-\frac{\lambda_{1}^{p-1} m_{0}^{2}}{2} \neq c_{4}$, since $\frac{v_{1}^{p+1}}{p+1}-\frac{\lambda_{1}^{p-1} v_{1}^{2}}{2}$ has only one minima for $v_{1} \in[0,+\infty]$. A contradiction is obtained. Similarly, we conclude that $r^{\alpha_{2}} u(r)$ approaches a finite limit as $r \rightarrow 0$.

Claim. (1) $\lim _{r \rightarrow \infty} v_{1}(r)$ must be either 0 or $\lambda_{1}$;

(2) $\lim _{r \rightarrow 0} v_{2}(r)$ must be either 0 or $\lambda_{2}$.

We only prove (1), the demonstration of (2) being the same. We denote the $\lim _{r \rightarrow \infty} v_{1}(r)$ by $v_{\infty}$. Now, if $v_{\infty} \neq 0$, we want to show that $v_{\infty}=\lambda_{1}$. From (2.5), we have

$$
\frac{d^{2} v_{1}}{d t^{2}}+\left(n-2-2 \alpha_{1}\right) \frac{d v_{1}}{d t}-\left(n-2-\alpha_{1}\right) \alpha_{1} v_{1}+v_{1}^{p}+e^{\left(l_{2}-(q-1) \alpha_{1}+2\right) t} v_{1}^{q}=0,
$$

where $t=\log r$. From Lemma 2.5, we have $v^{\prime}(t) \rightarrow 0$ as $t \rightarrow \infty$. So $\lim _{t \rightarrow \infty} v^{\prime \prime}(t)$ exists and must be 0 . Immediately, we have $v_{\infty}=\lambda_{1}$ or 0 .

If $\lim _{r \rightarrow \infty} v_{1}(r)=0$ or $\lim _{r \rightarrow 0} v_{2}(r)=0,(2.5)$ and (2.6) suggest that $v_{i}(i=$ $1,2)$ tend to zero at an algebraic rate. Indeed, since $p, q>1$ and $v_{i}(i=1,2)$ is expected to satisfy asymptotically the following equations

$$
\begin{gathered}
v_{1}^{\prime \prime}+\frac{n-1-2 \alpha_{1}}{r} v_{1}^{\prime}-\frac{\lambda_{1}^{p-1}}{r^{2}} v_{1}=0 \quad \text { as } \quad r \rightarrow \infty, \\
v_{2}^{\prime \prime}+\frac{n-1-2 \alpha_{2}}{r} v_{2}^{\prime}-\frac{\lambda_{2}^{q-1}}{r^{2}} v_{2}=0 \quad \text { as } r \rightarrow 0 .
\end{gathered}
$$

Therefore $v_{i}$ should satisfy the following asymptotical behaviors

$$
v_{1} \approx r^{-\left(n-2-\alpha_{1}\right)} \text { at } \infty, \quad v_{2} \approx r^{\alpha_{2}} \text { at } 0 .
$$

Now we claim: (i) If $\lim _{r \rightarrow \infty} v_{1}=0$, then $\lim _{r \rightarrow \infty} r^{n-2} u=c>0$;

(ii) If $\lim _{r \rightarrow 0} v_{2}=0$, then $\lim _{r \rightarrow 0} u=c_{1}>0$.

First, we prove (i), by assumption, for any $\varepsilon>0$ there exists a positive number $r_{\varepsilon}$ such that $v_{1}$ satisfies

$$
v_{1}^{\prime \prime}+\frac{n-1-2 \alpha_{1}}{r} v_{1}^{\prime}-\frac{\left(\lambda_{1}^{p-1}-\varepsilon\right)}{r^{2}} v_{1} \geq 0, r>r_{\varepsilon} .
$$

The characteristic equation of (3.11) has the two characteristic values

$$
\begin{aligned}
& a_{1}=\alpha_{1}-\frac{n-2-\sqrt{(n-2)^{2}-4 \varepsilon}}{2}=\alpha_{1}+O(\varepsilon), \\
& a_{2}=\alpha_{1}-\frac{n-2+\sqrt{(n-2)^{2}-4 \varepsilon}}{2}=\alpha_{1}+2-n+O(\varepsilon) .
\end{aligned}
$$

Rewrite (3.11) as

$$
\left(D-\frac{a_{1}-1}{r}\right)\left(D-\frac{a_{2}}{r}\right) v_{1} \geq 0
$$


where $D:=\frac{d}{d r}, D^{2}:=\frac{d^{2}}{d r^{2}}$.

Let $\left(D-\frac{a_{2}}{r} v_{1}\right)=U_{1}$, so we have

$$
U_{1}^{\prime}+\frac{1-a_{1}}{r} U_{1} \geq 0
$$

from which, we have

$$
\left[r^{1-\alpha_{1}+O(\varepsilon)}\left(D-\frac{a_{2}}{r}\right) v_{1}\right]^{\prime} \geq 0
$$

Observe that, for $\varepsilon$ small enough,

$$
\lim _{r \rightarrow \infty} r^{1-\alpha_{1}+O(\varepsilon)}\left(D-\frac{a_{2}}{r}\right) v_{1}=0 \quad \text { by }(2.9),
$$

since $1-\alpha_{1}+O(\varepsilon)<1$. It follows from (3.12) that

$$
\left(D-\frac{a_{2}}{r}\right) v_{1} \leq 0, \quad r>r_{\varepsilon} .
$$

Integrating once from $r_{\varepsilon}$ to $r$ yields

$$
v_{1} \leq c_{\varepsilon} r^{a_{2}}=c_{\varepsilon} r^{\alpha_{1}+2-n+O(\varepsilon)}, \quad r>r_{\varepsilon} .
$$

Thus for $\varepsilon$ sufficiently small,

$$
v_{1}^{\prime \prime}+\frac{n-1-2 \alpha_{1}}{r} v_{1}^{\prime}-\frac{\lambda_{1}^{p-1}}{r^{2}} v_{1}=g(r)
$$

with

$$
g(r)=\frac{v_{1}^{p}}{r^{2}}+r^{l_{2}-(q-1) \alpha_{1}} v_{1}^{q}=O\left(r^{-2-\delta}\right) \text { near } \infty,
$$

where $\delta$ is a positive constant.

Applying the method of variation of parameters to (3.13), $v_{1}$ is represented by

$$
\begin{aligned}
v_{1}(r)= & C_{1}(R) r^{\alpha_{1}}+C_{2}(R) r^{\alpha_{1}+2-n} \\
& +\frac{r^{\alpha_{1}+2-n}}{2-n} \int_{R}^{r} s^{n-1-\alpha_{1}} g(s) d s-\frac{r^{\alpha_{1}}}{2-n} \int_{R}^{r} s^{1-\alpha_{1}} g(s) d s .
\end{aligned}
$$

By a similar computation, we have, from (3.14) and the boundedness of $v_{1}(r)$,

$$
v_{1}(r)=C r^{\alpha_{1}+2-n}+o\left(r^{\alpha_{1}+2-n}\right),
$$

hence,

$$
r^{n-2} u(r) \leq C \text { at } r=\infty .
$$

By Lemma 2.6, there exists a constant $c$ such that $r^{n-2} u(r) \rightarrow c$ as $r \rightarrow \infty$.

The proof of (ii) is handled by the same way. As in the proof of (i), for any $\varepsilon^{\prime}>0$, there exists a positive number $r_{\varepsilon^{\prime}}$ such that

$$
\lim _{r \rightarrow 0} r^{n-1-\alpha_{2}+O\left(\varepsilon^{\prime}\right)}\left(D-\frac{\alpha_{2}}{r}\right) v_{2}=0,
$$

and

$$
\left(D-\frac{\alpha_{2}}{r}\right) v_{2} \geq 0, \quad 0<r<r_{\varepsilon^{\prime}} .
$$


Integrating once from $r$ to $r_{\varepsilon^{\prime}}$ yields

$$
v_{2} \leq c_{\varepsilon^{\prime}} r^{\alpha_{2}+O\left(\varepsilon^{\prime}\right)}
$$

Applying the method of variation of parameters to (2.6), we immediately obtain that $v_{2}$ is bounded by $r^{\alpha_{2}}$, and in turn $u$ is bounded, by standard theory, we have $\lim _{r \rightarrow 0} u(r)=c_{1}$.

When $p=\frac{n+2+2 l_{1}}{n-2}$ or $q=\frac{n+2+2 l_{2}}{n-2}$, there is another possibility: $r^{\frac{n-2}{2}} u$ could oscillate endlessly near $\infty$ or oscillate endlessly near the origin.

The proof of Theorem 1.4. (i) The function $v_{2}(t):=r^{\alpha_{2}} u, t=\log r$, satisfies

$$
v_{2}^{\prime \prime}-\lambda_{2}^{q-1} v_{2}+v_{2}^{q}+e^{\delta_{1} t} v_{2}^{p}=0
$$

where $\delta_{1}=\left(\alpha_{1}-\alpha_{2}\right)(p-1)>0$. Note that by Lemma 2.1(i), $v_{2}(t)$ is bounded near $-\infty$. Define an energy function

$$
E(t):=\frac{1}{2} v_{2}^{\prime 2}-\frac{\lambda_{2}^{q-1}}{2} v_{2}^{2}+\frac{1}{q+1} v^{q+1}
$$

As in (2.8), we have

$$
E(t)=C(T)+\int_{t}^{T} e^{\delta_{1} s} v_{2}^{p} v_{2}^{\prime} d s
$$

where $T$ is a fixed number.

In case that $v_{2}$ oscillates near $-\infty$, then we may suppose that

$$
0 \leq \mu_{1}=\lim _{t \rightarrow-\infty} \inf v_{2}(t)<\lim _{t \rightarrow-\infty} \sup v_{2}(t)=\mu_{2}<\infty .
$$

Then, there exist two sequences $\eta_{i}$ and $\varepsilon_{i}$ going to $-\infty$ as $i \rightarrow \infty$ such that $\eta_{i}$ and $\varepsilon_{i}$ are local minima and local maxima of $v_{2}$, respectively, satisfying $\eta_{i}<\varepsilon_{i}<\eta_{i+1}, i=1,2, \ldots$ From $(2.8)$, we know $\lim _{t \rightarrow-\infty} \int_{t}^{T} e^{\delta_{1} s} v_{2}^{p} v_{2}^{\prime} d s$ exists, so $E=\lim _{t \rightarrow-\infty} E(t)$ exists, and from which we have

$$
\lim _{i \rightarrow \infty} E\left(\eta_{i}\right)=-\frac{\lambda_{2}^{q-1}}{2} \mu_{1}^{2}+\frac{1}{q+1} \mu_{1}^{q+1}=-\frac{\lambda_{2}^{q-1}}{2} \mu_{2}^{2}+\frac{1}{q+1} \mu_{2}^{q+1}=\lim _{i \rightarrow \infty} E\left(\varepsilon_{i}\right),
$$

which implies that $b\left(\mu_{1}\right)=b\left(\mu_{2}\right)$.

Observe that for each $i>1$,

$$
0 \leq v_{2}^{\prime \prime}\left(\eta_{i}\right)=\lambda_{2}^{q-1} v_{2}\left(\eta_{i}\right)-v_{2}^{q}\left(\eta_{i}\right)-v_{2}^{p}\left(\eta_{i}\right) e^{\delta_{1} \eta_{i}},
$$

while

$$
0 \geq v_{2}^{\prime \prime}\left(\varepsilon_{i}\right)=\lambda_{2}^{q-1} v_{2}\left(\varepsilon_{i}\right)-v_{2}^{q}\left(\varepsilon_{i}\right)-v_{2}^{p}\left(\varepsilon_{i}\right) e^{\delta_{1} \varepsilon_{i}} .
$$

Putting $v_{2}\left(\eta_{i}\right)=\mu_{1, i}, v_{2}\left(\varepsilon_{i}\right)=\mu_{2, i}$, we have

$$
\lim _{i \rightarrow \infty} \mu_{1, i}=\mu_{1}, \quad \lim _{i \rightarrow \infty} \mu_{2, i}=\mu_{2} .
$$

From (3.16) and (3.17), we obtain

$$
0<\mu_{1} \leq \lambda_{2} \leq \mu_{2}
$$


The proof of (ii) can be handled in a similar way, we omit it here.

\section{A uniqueness result}

In this section, we shall prove a uniqueness result for positive radial singular solutions of (1.5) when $p>\frac{n+2+2 l_{1}}{n-2}$ and $q<\frac{n+2+2 l_{2}}{n-2}$. More precisely, we require that solutions be singular at infinity for $q<\frac{n+2+2 l_{2}}{n-2}$, and be singular at the origin for $p>\frac{n+2+2 l_{1}}{n-2}$. In order to prove Theorem 1.6, we need a finer asymptotic behavior of solution to (1.5) near 0 and $\infty$.

Let $w(t)=v_{1}-\lambda_{1}, t=\log r$. If $\lim _{t \rightarrow+\infty} v(t)=\lambda_{1}$, then we have $\lim _{t \rightarrow+\infty} w(t)=0$ and

(4.1) $w^{\prime \prime}+\left(n-2-2 \alpha_{1}\right) w^{\prime}+\left(2+l_{1}\right)\left(n-2-\alpha_{1}\right) w+f(w)+\left(w+\lambda_{1}\right)^{q} e^{\delta t}=0$, where $\delta=\frac{\left(2+l_{1}\right)(1-q)}{p-1}+2+l_{2}<0$, and

$$
\begin{aligned}
f(w) & =\left(\lambda_{1}+w\right)^{p}-\lambda_{1}^{p}-p \lambda_{1}^{p-1} w=\lambda_{1}^{p} \sum_{k=2}^{\infty} \frac{(p-k+1)}{k !}\left(\frac{w}{\lambda_{1}}\right)^{k} \\
& =\frac{(p-1) \lambda_{1}^{p-2}}{2} w^{2}+o\left(w^{2}\right) \text { for } w \text { near } 0 .
\end{aligned}
$$

Theorem 4.1. Let $u(r)$ be a singular solution of (1.5) at infinity and $\frac{n+l_{1}}{n-2}<$ $p<\frac{n+2+2 l_{1}}{n-2}$. Then for any $\varepsilon \in(0,-\delta)$ we have

$$
w(t)=O\left(e^{-\varepsilon t}\right), \quad w^{\prime}(t)=O\left(e^{-\varepsilon t}\right) \text { as } t \rightarrow+\infty .
$$

Proof. For $T>0$ and $t \in(0, T)$, multiplying (4.1) by $2 w^{\prime}(t)$ and integrating from $t$ to $T$, we obtain

$$
\begin{aligned}
& {\left.\left[w^{\prime 2}+\left(2+l_{1}\right)\left(n-2-\alpha_{1}\right) w^{2}\right]\right|_{t} ^{T}+2\left(n-2-2 \alpha_{1}\right) \int_{t}^{T} w^{\prime 2} d s} \\
& +2 \int_{t}^{T} f(s) w^{\prime} d s+2 \int_{t}^{T}\left(w+\lambda_{1}\right)^{q} e^{\delta t} w^{\prime} d s=0 .
\end{aligned}
$$

By (2.10), it is easy to see that for large $t$,

$$
\begin{aligned}
& w^{\prime 2}(T) \rightarrow 0, w^{2}(T) \rightarrow 0, \\
& \int_{t}^{T} f(s) w^{\prime} d s=-\frac{(p-1) \lambda_{1}^{p-2}}{6} w^{3}(t)+o\left(w^{3}(t)\right) \text { as } T \rightarrow+\infty .
\end{aligned}
$$

It follows from Lemma 2.4 and (4.3) (letting $T \rightarrow \infty$ ) that, for large $t$,

$$
w^{\prime 2}(t)+\left(2+l_{1}\right)\left(n-2-\alpha_{1}\right) w^{2}(t) \leq C|w|^{3}+\int_{t}^{\infty}\left(w+\lambda_{1}\right)^{q} e^{\delta s} w^{\prime} d s,
$$

since $n-2-2 \alpha_{1}<0$. It follows that for large $t$

$$
w^{\prime 2}(t)+\left(2+l_{1}\right)\left(n-2-\alpha_{1}\right) w^{2}(t) \leq C \int_{t}^{\infty}\left(w+\lambda_{1}\right)^{q} e^{\delta s} w^{\prime} d s \leq C e^{\delta t}
$$


Hence by (4.4) and the fact $\left(2+l_{1}\right)\left(n-2-2 \alpha_{1}\right)>0$, we have

$$
\left|w^{\prime}(t)\right|+|w(t)| \leq C e^{\frac{\delta}{2} t}
$$

Therefore

$$
\left|\int_{t}^{T}\left(w+\lambda_{1}\right)^{q} e^{\delta t} w^{\prime} d s\right| \leq C \int_{t}^{T} e^{\frac{3 \delta}{2} s} \leq C e^{\frac{3 \delta}{2} t},
$$

which, together with (4.4), imply, that

$$
\left|w^{\prime}(t)\right|+|w(t)| \leq C e^{\frac{3 \delta}{4} t} .
$$

Thus for any $m>0$, using a simple iteration of $m$-step in (4.4) yields

$$
\left|w^{\prime}(t)\right|+|w(t)| \leq C e^{\frac{\left(2^{m}-1\right) \delta}{2^{m}} t}
$$

and (4.2) follows by taking $m$ large.

Remark 4.2. As a matter of fact, applying the method of variation of parameters to (4.1), we have that $\varepsilon$ can reach at $-\delta$. Because of (4.2), we have $f(t)=O\left(w^{2}(t)\right)=O\left(e^{\delta t}\right)$ and

$$
w(t)=-\frac{1}{\omega} \int_{t}^{\infty} e^{\frac{n-2-2 \alpha_{1}}{2}(s-t)} \sin \omega(s-t)\left[f(s)+e^{\delta t}\left(v_{1}+\lambda_{1}\right)^{p}\right] d s=O\left(e^{\delta t}\right),
$$

where $\omega=\left(\left(2+l_{1}\right)\left(n-2-\alpha_{1}\right)-\frac{1}{4}\left(n-2-2 \alpha_{1}\right)^{2}\right)^{\frac{1}{2}}$.

Remark 4.3. If $u(r)$ is a singular solution of (1.5) at 0 , and $\frac{n+l_{1}}{n-2}<q<\frac{n+2+2 l_{2}}{n-2}$, then we have a similar result

$$
v_{2}(r)-\lambda_{2}=O\left(r^{\epsilon}\right), \quad\left(v_{2}(r)-\lambda_{2}\right)^{\prime}=O\left(r^{\epsilon+1}\right) \text { as } r \rightarrow 0,
$$

where $\epsilon \in\left(0, \frac{\left(2+l_{2}\right)(1-p)}{q-1}+2+l_{1}\right)$.

Now we are ready to prove Theorem 1.6. Let $u_{1}(r)$ and $u_{2}(r)$ be two different singular solutions of (1.5) at infinity. We introduce the function

$$
\bar{w}(t)=r^{\alpha_{1}} u_{1}(r)-r^{\alpha_{1}} u_{2}(r)=w_{1}(t)-w_{2}(t), \quad t=\log r,
$$

and show that $\bar{w}(t)$ is identical to zero.

Proof of Theorem 1.6. Clearly $\bar{w}(t)$ satisfies

$$
\begin{aligned}
& \bar{w}^{\prime \prime}(t)+\left(n-2-2 \alpha_{1}\right) \bar{w}^{\prime}(t)+\left(2+l_{1}\right)\left(n-2-\alpha_{1}\right) \bar{w} \\
& +\left(f\left(w_{1}\right)-f\left(w_{2}\right)\right)+\left[\left(w_{1}(t)+\lambda_{1}\right)^{q}-\left(w_{2}(t)+\lambda_{1}\right)^{q}\right] e^{\delta t}=0 .
\end{aligned}
$$

For $T>0$ and $t \in(0, T)$, multiplying (4.5) by $2 \bar{w}^{\prime}$ and integrating from $t$ to $T$, we obtain

$$
\begin{aligned}
& {\left.\left[\bar{w}^{\prime 2}+\left(2+l_{1}\right)\left(n-2-\alpha_{1}\right) \bar{w}^{2}\right]\right|_{t} ^{T}+\left(n-2-2 \alpha_{1}\right) \int_{t}^{T} \bar{w}^{\prime 2}} \\
& +2 \int_{t}^{T}\left[f\left(w_{1}\right)-f\left(w_{2}\right)\right] \bar{w}^{\prime}+2 \int_{t}^{T}\left[\left(w_{1}(t)+\lambda_{1}\right)^{q}-\left(w_{2}(t)+\lambda_{1}\right)^{q}\right] e^{\delta s} \bar{w}^{\prime}=0 .
\end{aligned}
$$


Thus we have the following estimates:

$$
\left|\int_{t}^{T}\left[f\left(w_{1}\right)-f\left(w_{2}\right)\right] \bar{w}^{\prime}\right| \leq C \int_{t}^{T}\left|\bar{w}^{\prime} \bar{w}\right| e^{-\varepsilon s} \leq C \int_{t}^{T} e^{-\varepsilon s}\left(\bar{w}^{2}+\bar{w}^{\prime 2}\right)
$$

and

$$
\int_{t}^{T}\left[\left(w_{1}(t)+\lambda_{1}\right)^{q}-\left(w_{2}(t)+\lambda_{1}\right)^{q}\right] e^{\delta s} \bar{w}^{\prime} \leq C \int_{t}^{T} e^{\delta s}\left(\bar{w}^{2}+\bar{w}^{\prime 2}\right) .
$$

For large $t$, it follows, by letting $T \rightarrow+\infty$ and using Theorem 4.1, that

$$
\bar{w}^{\prime 2}(t)+\left(2+l_{1}\right)\left(n-2-\alpha_{1}\right) \bar{w}^{2}(t) \leq C \int_{t}^{\infty} e^{-\varepsilon s}\left(\bar{w}^{2}+\bar{w}^{\prime 2}\right),
$$

since $n-2-2 \alpha_{1} \leq 0$. From which, we have

$$
\bar{w}^{\prime 2}(t)+\bar{w}^{2}(t) \leq C \int_{t}^{\infty} e^{-\varepsilon s}\left(\bar{w}^{2}+\bar{w}^{\prime 2}\right)
$$

sine $\left(2+l_{1}\right)\left(n-2-\alpha_{1}\right)>0$. Hence $\bar{w}^{2}(t)+\bar{w}^{2}(t) \equiv 0$ for all sufficiently large $t$ by the Gronwall inequality. According to the uniqueness of ordinary differential equation, $w$ is identically zero for all $t$.

When $p>\frac{n+2+2 l_{1}}{n-2}$, we use the transformation

$$
\bar{w}_{1}(t)=r^{\alpha_{2}} u_{1}(r)-r^{\alpha_{2}} u_{2}(r)=z_{1}(t)-z_{2}(t), \quad t=\log r,
$$

where $z_{i}(t)=r^{\alpha_{2}} u_{i}(r)-\lambda_{2} \rightarrow 0(i,=1,2)$ as $t \rightarrow-\infty$. Then $\bar{w}_{1}(t)$ satisfies

$$
\begin{aligned}
& \bar{w}_{1}^{\prime \prime}(t)+\left(n-2-2 \alpha_{2}\right) \bar{w}_{1}{ }^{\prime}(t)+\left(2+l_{2}\right)\left(n-2-\alpha_{2}\right) \bar{w}_{1} \\
& +\left(\bar{f}\left(z_{1}\right)-\bar{f}\left(z_{2}\right)\right)+\left[\left(z_{1}(t)+\lambda_{2}\right)^{p}-\left(z_{2}(t)+\lambda_{2}\right)^{p}\right] e^{\delta_{2} t}=0,
\end{aligned}
$$

where $\delta_{2}=(p-1)\left(\alpha_{1}-\alpha_{2}\right)>0$, and

$$
\begin{aligned}
\bar{f}(z) & =\left(\lambda_{2}+z\right)^{q}-\lambda_{2}^{q}-q \lambda_{2}^{q-1} z=\lambda_{2}^{q} \sum_{k=2}^{\infty} \frac{q-k+1}{k !}\left(\frac{z}{\lambda_{2}}\right)^{k} \\
& =\frac{(q-1) \lambda_{2}^{q-2}}{2} z^{2}+o\left(z^{2}\right) \text { for } t \text { near }-\infty .
\end{aligned}
$$

The equation (4.7) is the same as (4.5). On the other hand, one can easily see from previous proof that the key ingredient of the proof for the case $p>\frac{n+2+2 l_{1}}{n-2}$ is that the coefficient of the term $\bar{w}_{1}{ }^{\prime}(t)$ is not less than 0 . Fortunately, we have $n-2-2 \alpha_{2}>0$ when $p>\frac{n+2+2 l_{1}}{n-2}$. Hence the proof above carries over immediately.

Acknowledgements. The authors are greatly indebted to Professor Yi Li for many useful discussions, suggestions and comments. 


\section{References}

[1] S. Bae, Positive entire solutions of semilinear elliptic equations with quadratically vanishing coefficient, J. Differential Equations 237 (2007), no. 1, 159-197.

[2] _ Separation structure of positive radial solutions of a semilinear elliptic equation in $\mathbb{R}^{n}$, J. Differential Equations 194 (2003), no. 2, 460-499.

[3] S. Bae, T.-K. Chang, and D.-H. Pank, Infinite multiplicity of positive entire solutions for a semilinear elliptic equation, J. Differential Equations 181 (2002), no. 2, 367-387.

[4] S. Bae and W.-M. Ni, Existence and infinite multiplicity for an inhomogeneous semilinear elliptic equation on $\mathbb{R}^{n}$, Math. Ann. 320 (2001), no. 1, 191-210.

[5] R. Bamón, I. Flores, and M. del Pino, Ground states of semilinear elliptic equations: a geometric approach, Ann. Inst. H. Poincare Anal. Non Lineaire 17 (2000), no. 5, $551-581$.

[6] G. Bernard, An inhomogeneous semilinear equation in entire space, J. Differential Equations 125 (1996), no. 1, 184-214.

[7] Y.-B. Deng, Y. Li, and Y. Liu, On the stability of the positive radial steady states for a semilinear Cauchy problem, Nonlinear Anal. 54 (2003), no. 2, 291-318.

[8] Y.-B. Deng, Y. Li, and F. Yang, On the stability of the positive steady states for a nonhomogeneous semilinear Cauchy problem, J. Differential Equations 228 (2006), no. 2, 507-529.

[9] C.-F. Gui, Positive entire solutions of the equation $\Delta u+f(x, u)=0$, J. Differential Equations 99 (1992), no. 2, 245-280.

[10] - On positive entire solutions of the elliptic equation $\Delta u+K(x) u^{p}=0$ and its applications to Riemannian geometry, Proc. Roy. Soc. Edinburgh Sect. A 126 (1996), no. 2, 225-237.

[11] C.-F. Gui, W.-M. Ni, and X.-F. Wang, On the stability and instability of positive steady states of a semilinear heat equation in $\mathbb{R}^{n}$, Comm. Pure Appl. Math. 45 (1992), no. 9, 1153-1181.

[12] _ Further study on a nonlinear heat equation, J. Differential Equations 169 (2001), no. 2, 588-613.

[13] K. Hayakawa, On nonexistence of global solutions of some semilinear parabolic differential equations, Proc. Japan Acad. 49 (1973), 503-505.

[14] D. D. Joseph and T. S. Lundgren, Quasilinear Dirichlet problems driven by positive sources, Arch. Rational Mech. Anal. 49 (1972), 241-269.

[15] T.-Y. Lee and W.-M. Ni, Global existence, large time behavior and life span of solutions of a semilinear parabolic Cauchy problem, Trans. Amer. Math. Soc. 333 (1992), no. 1, 365-378.

[16] Y. Li, Remarks on a semilinear elliptic equation on $\mathbb{R}^{n}$, J. Differential Equations $\mathbf{7 4}$ (1988), no. 1, 34-49.

[17] Asymptotic behavior of positive solutions of equation $\Delta u+K(x) u^{p}=0$ in $\mathbb{R}^{n}$, J. Differential Equations 95 (1992), no. 2, 304-330.

[18] Y. Li and W.-M. Ni, On conformal scalar curvature equations in $\mathbb{R}^{n}$, Duke Math. J. 57 (1988), no. 3, 895-924.

[19] Y. Liu, Y. Li, and Y.-B. Deng, Separation property of solutions for a semilinear elliptic equation, J. Differential Equations 163 (2000), no. 2, 381-406.

[20] W.-M. Ni, On the elliptic equation $\Delta u+K(x) u^{(n+2) /(n-2)}=0$, its generalizations, and applications in geometry, Indiana Univ. Math. J. 31 (1982), no. 4, 493-529.

[21] W.-M. Ni and S. Yotsutani, Semilinear elliptic equations of Matukuma-type and related topics, Japan J. Appl. Math. 5 (1988), no. 1, 1-32.

[22] J. Serrin and H. Zou, Classification of positive solutions of quasilinear elliptic equations, Topol. Methods Nonlinear Anal. 3 (1994), no. 1, 1-25.

[23] X.-F. Wang, On the Cauchy problem for reaction-diffusion equations, Trans. Amer. Math. Soc. 337 (1993), no. 2, 549-590. 
[24] F. Weissler, Existence and nonexistence of global solutions for a semilinear heat equation, Israel J. Math. 38 (1981), no. 1-2, 29-40.

[25] E. Yanagida and S. Yotsutani, Classification of the structure of positive radial solutions to $\Delta u+K(|x|) u^{p}=0$ in $\mathbb{R}^{n}$, Arch. Rational Mech. Anal. 124 (1993), no. 3, 239-259.

BAISHUN LAI

Institute of Contemporary Mathematics

HENAN UNIVERSITY

AND

SCHOOL OF Mathematics

Henan University

Kaifeng, Henan 475004, P. R. China

E-mail address: laibaishun@henu.edu.cn

QING LUO

SCHOOL OF MATHEMATICS

HENAN UNIVERSITY

Kaifeng, Henan 475004, P. R. China

E-mail address: Lq@henu.edu.cn

SHUQING ZHOU

School of Mathematics and Computer Science

Hunan Normal University

Changsha, Hunan 410081, P. R. China

E-mail address: zhoushuqing87@163.com 